\title{
Performance Degradation Due to Code Tracking Errors in Spread-Spectrum Code-Division Multiple-Access Systems
}

\author{
Bas W.'t. Hart, Student Member, IEEE, Richard D. J. Van Nee, Member, IEEE, \\ and Ramjee Prasad, Senior Member, IEEE
}

\begin{abstract}
The influence of code synchronization errors on the performance of direct-sequence spread-spectrum (DS/SS) communication systems is investigated. Insight is gained in the degradation of some basic performance parameters due to the tracking bias of a noncoherent delay lock loop (DLL). The performance parameters investigated are the bit-error probability, throughput and delay. Numerical results show that for receivers with an early-late spacing of $d=1$, using a noncoherent DLL, the results of a system performance analysis in a fast fading environment can be much too optimistic if the tracking errors are ignored.
\end{abstract}

\section{INTRODUCTION}

$\mathbf{C}$ ODE-division multiple-access (CDMA) is a strong candidate for the choice of multiple-access techniques for future wireless personal communication systems and networks, and for several years now, it is an important research topic. There are several publications, e.g., [1]-[9], on the performance analysis of systems and networks using spreadspectrum CDMA techniques. In these papers, it was assumed that the influence of code synchronization errors on the system performance caused by multipath and multiuser/transmitter interference could be ignored. However, this assumption is valid only for specific conditions. Most papers on spreadspectrum synchronization errors due to multipath propagation and interference, e.g., [10], focus on ranging and timing applications. For communication applications, however, the situation for code tracking is different. In the case of ranging or timing, we want to track the delay of the line-of-sight (LOS) signal; for code tracking in communication applications, the key parameter is the delay that maximizes the correlation output.

Fig. 1 shows an example of a correlation function of an input signal consisting of two multipath components with a relative spacing smaller than one chip time. The output correlation function is drawn for the cases of in-phase and out-of-phase signals.

Due to fading, the peak value of the correlation function and the delay that maximizes the correlation output vary significantly (in the case of Fig. 1 the received signal varies

\footnotetext{
Manuscript received April 27, 1995; revised October 4, 1995. This paper was presented in part at the 19956 th IEEE International Symposium on Personal, Indoor, and Mobile Radio Communications, Toronto, Canada, September 1995.

The authors are with the Telecommunications and Traffic Control Systems Group, Delft University of Technology, The Netherlands.

Publisher Item Identifier S 0733-8716(96)05215-8.
}

between the curves $c$ and $d$ ). In order to minimize the loss of signal power, the code tracking loop should track the instantaneous delay of the correlation function, which requires the loop bandwidth to be much larger than the fading bandwidth. As long as the fading bandwidth is smaller than the loop bandwidth (slow fading), the assumption that the influence of the synchronization errors due to fading on the system performance can be ignored, is valid. However, there is an upper limit to the tracking loop bandwidth: an increase in the loop bandwidth also causes the loop noise to increase. For a carrier tracking loop, the calculation of the optimal loop bandwidth, which minimizes the total effect of both noise and fading, is described in [11]. For a tracking loop bandwidth smaller than the fading bandwidth, synchronization errors occur: a delay error is caused because the tracking loop is not able to track the instantaneous delay. As will be explained in the next section, by using a noncoherent delay lock loop (DLL) to estimate the code delay, a tracking bias is introduced in a fast fading environment. This bias can have a considerable impact on the system performance. Little is known about the influence of these code tracking errors on the performance of CDMA communication systems. In this paper, insight is gained in the performance degradation of a direct sequence spread-spectrum system with binary phase shift keying (BPSK) modulation due to the tracking bias of a noncoherent delay lock loop. The technique of noncoherent DLL, which is used by many receivers, is close to optimum for code tracking in an additive white Gaussian noise (AWGN) channel, but not in a multipath or multiuser channel (as in CDMA). This DLL is specifically designed for the AWGN channel; multipath or multiuser interference either enhances the noise level, or introduces tracking biases as described in this paper and in [10], [12], and [13]. We have analyzed the performance of conventional direct sequence receivers with noncoherent delay lock loops. No channel estimation is involved, other than the phase and delay estimation of the delay lock loop. Rician channels are considered for mobile satellite links and Rayleigh for mobile terrestrial systems.

Two fading models are used for the performance degradation analysis: the model of an unfiltered input signal consisting of a LOS or otherwise dominant path component and just one multipath signal, and the model of an unfiltered input signal consisting of a LOS (or dominant path) component and multiple multipath signals. For both models the correlation 


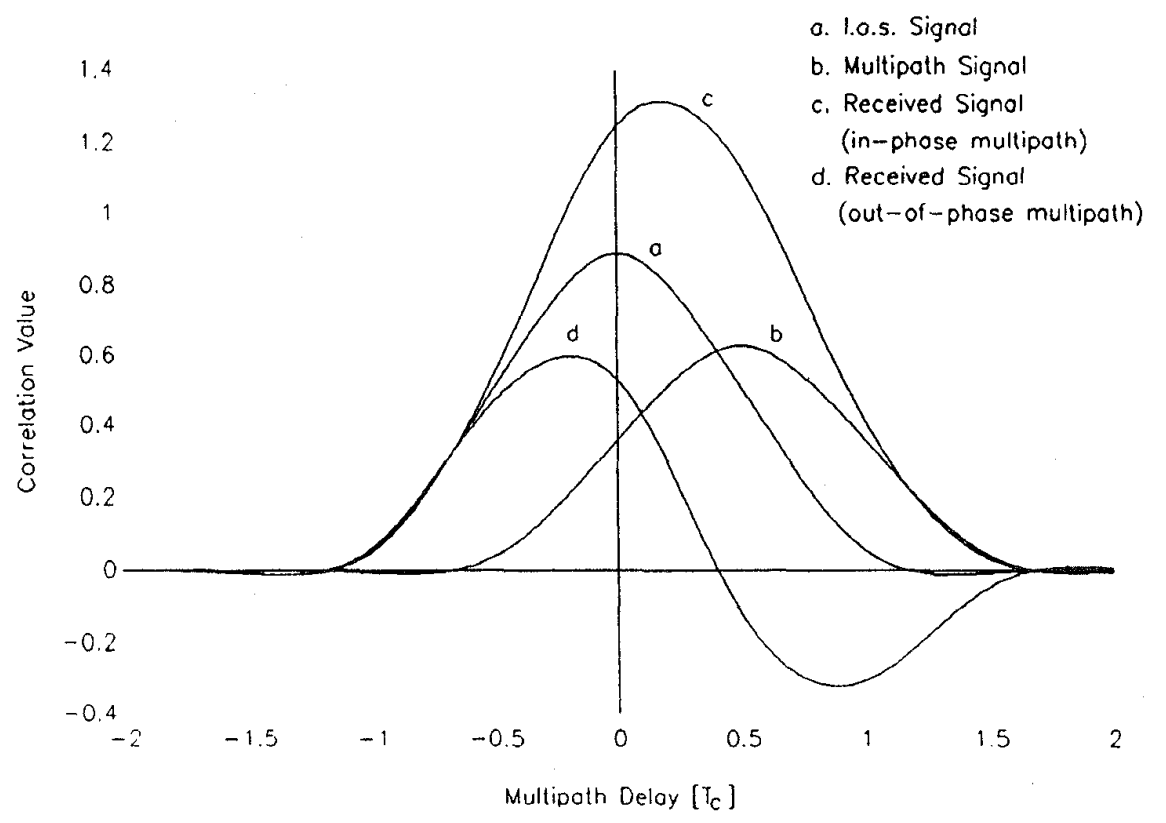

Fig. 1. Correlation function with in-phase and out-of-phase multipath signals.

output is determined from the calculated tracking bias. This is done in a deterministic way for the single multipath case. For the multiple multipath case, a Gaussian distribution for the correlation output is assumed. With the correlation output the bit-error probability is obtained, which gives an indication of the influence of synchronization errors on the system performance in case of circuit-switched mobile communications. Because of the recent interest in the use of CDMA for packetswitched radio networks, it is important to study the effects of tracking errors on the throughput and the delay. The system performance parameters calculated with tracking bias have been compared to the performance parameters determined when this error is ignored. In these analyses, the relative levels of performance are far more important than the absolute levels. The research does not apply to satellite based systems only, but to CDMA systems in a fast fading environment in general.

In Section II, the synchronization errors due to multipath fading and multitransmitter interference are discussed. Section III deals with the most important performance parameters. First, the bit-error probability and the packet success probability are discussed. After that, the throughput and the delay are considered. In Section IV, an analysis with numerical results is given on the single multipath signal case and on the multiple multipath case. Possible remedies for the effects of tracking errors are discussed in Section V. Conclusions are given in Section VI.

\section{Code Tracking ERrors}

\section{A. Code Tracking Errors Due to Multipath Propagation}

The received signal can be written as

$$
r(t)=\sum_{i=0}^{M} a_{i}(t)\left[t-\tau_{i}(t)\right] b\left[t-\tau_{i}(t)\right] \cos \left[\omega t+\theta_{i}(t)\right]
$$

where $M$ is the number of multipath signals that are taken into account, $p(t)$ is the spread-spectrum code, $b(t)$ is the data signal, $\omega$ is the angular frequency of the LOS signal and $a_{i}(t), \tau_{i}(t)$, and $\theta_{i}(t)$ are the time dependent amplitude, delay, and phase of the $i$ th signal, respectively. From now on, for simplicity of notation, the time dependence of the parameters $a_{i}, \tau_{i}$, and $\theta_{i}$ is left out. Noise and multitransmitter interference are left out in (1), since the primary interest here is the influence of multipath fading on synchronization errors. The data signal $b(t)$ has not be used in the remainder of this section since its influence on tracking is removed by envelope detection in a noncoherent DLL.

Notice that in general, all signals can have different frequencies $\left[\omega+\delta \theta_{i}(t) / \delta t\right] / 2$. The bandwidth spread of these frequencies is called the fading bandwidth, $B_{F}$, which is a crucial parameter in the analysis of multipath tracking errors. The fading bandwidth depends on the change of the transmitter-reflector-receiver geometry. If, for instance, the receiver velocity is $10 \mathrm{~m} / \mathrm{s}$, the fading bandwidth becomes $100 \mathrm{~Hz}$ for a carrier frequency of $1.5 \mathrm{GHz}$.

In order to track the desired signal delay $\tau_{d}$, for communication applications the delay that maximizes the correlation output, the input signal (1) is down-converted and correlated with an "early" and a "late" code. These are replicas of the received spread spectrum code with a delay of plus or minus $d T_{c} / 2$ seconds compared to a "prompt" code, respectively. $T_{c}$ is the chip time and the parameter $d$ is often referred to as the early-late spacing. For communication applications, $d=1$ is a typical value.

In the case of a noncoherent DLL, the resulting early and late correlation functions are first squared and then subtracted to produce the " $S$-curve" $S_{\mathrm{nc}}(\tau)$ thereby removing the influ- 
ence of the data and the carrier phase

$$
\begin{aligned}
S_{\mathrm{nc}}(\hat{\tau})= & \left\|\sum_{i=0}^{M} a_{i} R\left(\hat{\tau}-\tau_{i}+\frac{d}{2} T_{c}\right) \exp \left(j \theta_{i}\right)\right\|^{2} \\
& -\left\|\sum_{i=0}^{M} a_{i} R\left(\hat{\tau}-\tau_{i}-\frac{d}{2} T_{c}\right) \exp \left(j \theta_{i}\right)\right\|^{2}
\end{aligned}
$$

where $R(\tau)$ is the correlation function of the spread-spectrum code and $\hat{\tau}$ is the estimate of the code delay. For an unfiltered ideal code, $R(\tau)$ is equal to $1-\left|\tau / T_{c}\right|$ for $|\tau| \leq T_{c}$ and equal to zero elsewhere. The DLL tracks that value of $\hat{\tau}$ for which $S_{\mathrm{nc}}(\hat{\tau})$ is zero while its slope $\delta S_{\mathrm{nc}}(\hat{\tau}) / \delta \hat{\tau}$ is negative.

The carrier tracking loop tracks the phase of the received signal after correlation with the prompt code. The carrier phase estimate can be expressed as

$$
\hat{\theta}=\arg \left[\sum_{i=0}^{M} a_{i} \exp \left(j \theta_{i}\right) R\left(\hat{\tau}-\tau_{i}\right)\right] .
$$

To understand the effects of multipath propagation on code tracking, it is important to distinguish two different cases: the fading bandwidth $B_{F}$ is large compared to the tracking loop bandwidth $B_{L}$ or $B_{F}$ is small compared to $B_{L}$. For a noncoherent DLL also the predetection bandwidth $B_{p}$, which has to be larger than or equal to the data bandwidth in order to let the signal pass, has to be considered. In the slow fading case, $B_{F}$ is small compared to $B_{L}$ and also to $B_{p}$, since $B_{p}$ is always larger than $B_{L}$. If $B_{F}$ is large compared to $B_{L}$, but small compared to $B_{p}$, all multipath signals pass the predetection correlation. This is fast fading. Finally, there can be a second type of fast fading for which $B_{F}$ is large in comparison to both $B_{L}$ and $B_{p}$.

1) Slow Fading: If $B_{F}$ is small compared to $B_{L}$, then the averaging in the delay lock loop has no influence on the tracking of the delay of the summed LOS and multipath signals; the DLL simply locks on the value of $\tau_{d}$. For a small $B_{F} / B_{L}$ ratio the receiver will "see" the instantaneous delay and no tracking errors due to multipath fading occur.

2) Fast Fading: If $B_{F}$ is large compared to $B_{L}$, the noncoherent delay lock loop tracks the zero crossings of the time averaged $S$-curve. In case of fast fading when all multipath signals pass the predetection correlation, the time average is, because of the squaring operations in (2), equal to

$$
\begin{aligned}
S_{\mathrm{nc}}(\hat{\tau})= & \sum_{i=0}^{M}\left[a_{i} R\left(\hat{\tau}-\tau_{i}+\frac{d}{2} T_{c}\right)\right]^{2} \\
& -\left[a_{i} R\left(\hat{\tau}-\tau-\frac{d}{2} T_{c}\right)\right]^{2} .
\end{aligned}
$$

All cross products are filtered out because of their relatively high frequencies. The resulting $S$-curve is simply the summation of $M+1$ different noncoherent $S$-curves, which causes a certain tracking bias compared to the LOS signal delay, $\tau_{0}$, that is always positive. Due to fading, the instantaneous delay varies around $\tau_{0}$. For a positive instantaneous delay (e.g., curve $c$ in Fig. 1), the difference between $\tau_{d}$ and the biased estimated delay will be small. In the case of a negative value of the instantaneous delay (e.g., curve $d$ in Fig. 1) however, the tracking bias leads to a considerable tracking error and therefore to a substantial loss of signal power.

In the second case of fast fading, when $B_{F}$ is large compared to both $B_{L}$ and $B_{p}$, the noncoherent DLL will lock onto the dominant path, because the multipath signals cannot pass the predetection correlation. The tracking error will be small because no tracking bias is introduced.

As explained above, there is no tracking bias if $B_{F}$ is large compared to both $B_{L}$ and $B_{p}$. However, in practice, $B_{F}$ will be in the order of $100 \mathrm{~Hz}$ or less, while $B_{P}$ has to be larger or equivalent to the data bandwidth, which will generally be larger than $100 \mathrm{~Hz}$. So, values of $B_{F}$ that are much larger than $B_{P}$ are not very likely.

Also for $B_{F}<B_{L}$, the slow fading case, there will be no tracking errors due to multipath fading. Problems occur for systems for which the tracking loop bandwidth is smaller than $100 \mathrm{~Hz}$. As a rule of thumb, $B_{L}$ has to be about $1 \%$ of the symbol rate in order to get an acceptable tracking error and mean time to loose lock. Thus, systems with symbol rates less than $10 \mathrm{kBaud}$ can encounter problems because of tracking biases. In case of broadband CDMA applications (assuming a symbol rate higher than $10 \mathrm{kBaud}$ ) no multipath problems will occur, contrary to current CDMA communication systems with a bit rate of 9600 bps and systems like GPS with a 50 bps data signal.

So, tracking errors due to multipath will occur in a fast fading environment as defined above. Therefore, this situation has been considered in this paper in analyzing the performance degradation due to the multipath tracking problems.

\section{B. Code Tracking Errors Due to Multitransmitter Interference}

It was shown in [10] and [13] that tracking errors caused by multitransmitter or multiuser interference can be modeled in a similar way as those caused by multipath. The main difference is that multiuser interference can cause both positive and negative biases, because interfering cross-correlation peaks can have positive and negative delays relative to the desired correlation peak, while multipath correlation peaks only have positive delays (neglecting autocorrelation sidelobes). Another difference is that the phase of multiuser interference can change every symbol, so that it has the same effect as multipath interference with a fading bandwidth equal to the symbol rate. Thus, one interfering user causes tracking errors which are comparable to multipath tracking errors with the signal-to-multipath ratio (SMR) substituted by the signal-tointerference ratio (SIR). In the presence of a number of interferers, the variance of the resulting tracking error may be approximated as the sum of variances caused by all individual interferers. Based on the results in Section IV, the tracking error standard deviation caused by one interferer will be in the order of $\mathrm{SIR}^{-1}$. For $K$ users, this gives a standard deviation of $\sqrt{ } K \cdot \mathrm{SIR}^{-1}$. Since for the cases of interest $K<N$ and $\mathrm{SIR} \cong N(N$ is the code length $)$, the resulting tracking error can be expected to be less than $\sqrt{ } N / N$. For practical values of $N$ larger than 100, this gives tracking errors less than one tenth of a chip, which is negligible compared to the tracking 
errors caused by multipath, as demonstrated further on in this paper.

\section{PERFormanCE ANALYSIS}

This section presents a performance analysis of a direct sequence spread-spectrum CDMA system with BPSK modulation, in terms of bit-error probability, packet success probability, throughput and delay.

\section{A. Bit-Error Probability}

Assuming that the data bits -1 and 1 are equiprobable, the bit-error probability $P_{e}$ can be expressed as the probability that the correlation output $z$ is negative while the transmitted data bit was positive

$$
P_{e}=P(z<0 \mid b=1) \text {. }
$$

In general, in the case of Gaussian noise $P_{e}$ can be written as

$$
P_{e}=\int_{-\infty}^{\infty} p_{e}(x A) p_{u}(x) d x
$$

where $p_{u}(x)$ represents the probability density function of the desired part of the correlation output $z$ (see [2]) and $p_{e}(x A)$ the bit-error probability, given a signal amplitude $A$ and a factor $x$ caused by the path gain and by the signal loss due to tracking errors. $p_{e}(x A)$ is given by

$$
p_{e}(x A)=\frac{1}{2} \operatorname{erfc}\left(\frac{x A}{\sigma \sqrt{2}}\right)
$$

where $\operatorname{erfc}(x)$ is the complementary error function and $\sigma^{2}$, the total variance of the Gaussian noise, the sum of the noise variance $N_{0} / T_{b}$ and the interference power $\sigma_{i}^{2}$.

\section{B. Packet Success Probability}

Knowing the bit-error probability it is possible to calculate the packet success probability, which is necessary to obtain the throughput and delay values of CDMA packet transmissions. Because of our interests in the influence of synchronization errors, the packet success probability $P_{s}$ has been evaluated for fast fading only. In this case, it is assumed that the path gains are uncorrelated for two consecutive data bits. If packets of $N_{p}$ bits are transmitted, using a forward error correcting code that can correct up to $t$ errors per packet, then the packet success probability in the case of fast fading is

$$
P_{s}=\sum_{j=0}^{t} P_{e}^{j}\left(1-P_{e}\right)^{N_{p}-j}\left(\begin{array}{c}
N_{p} \\
j
\end{array}\right) .
$$

\section{Throughput}

Consider a communication network with a random access slotted CDMA scheme, where the data sequence is spread by a certain spreading code, consisting of $N$ chips per bit. The code length and the total number of codes may be fixed to $N$, but it may also be larger than $N$. It is assumed that the codes can be approximated by random sequences. Another assumption is, that the total number of users is large enough to get a Poisson distribution function for the offered traffic. Then, the probability $P_{\text {tr }}(k)$ that $k$ packets are generated during a certain time slot is given by

$$
P_{\mathrm{tr}}(k)=\frac{G^{k}}{k !} \exp (-G) .
$$

Here, $G$ is the average number of transmitted packets per time slot.

When a packet is transmitted, there is a certain success probability $P_{s}$ that it is received correctly. It is assumed that an acknowledgment is sent after successful reception of a packet, so after waiting twice the propagation delay, a user knows if its packet is received or not. Although a receiver itself could estimate whether a packet was successfully received in a single cell or single spot system, acknowledgments are mandatory for multicell or multispot systems, at least if a zero packet loss probability is required. When the transmitting user does not receive an acknowledgment for a certain packet, it retransmits that packet after a certain random delay. The steady state throughput of this transmission system is defined as the average number of successfully received packets per time slot, given by

$$
S=\sum_{k=1}^{K_{\max }} k P_{\mathrm{tr}}(k) P_{s}
$$

Here, $K_{\max }$ is the maximum number of users that can be simultaneously handled by the system, because the number of receivers or available code words is limited.

\section{Delay}

The corresponding average packet delay $D$ is defined as the number of slot times it takes for a packet to be successfully received. Thus it is the average time duration (in slots) between the packet being offered to the transmitter and the packet being successfully received [4], and is given by

$$
D=1.5+T_{d}+\left[\frac{G}{S}-1\right]\left[\frac{N_{\mathrm{AT}}}{2}+1+2 T_{d}\right]
$$

where $G / S-1$ is the average number of retransmissions, $N_{\text {AT }} / 2$ is the mean retransmission delay and $T_{d}$ is the propagation delay.

\section{Computational Results}

In this section computational results are given on performance parameters for the case that the code tracking error is ignored and for the case that the code tracking error is taken into account. The accent will be on the comparison of the two cases.

\section{A. Single Multipath Signal}

First, the situation of an unfiltered input signal consisting of a LOS component and just one multipath signal is considered. We assume only one user, so there is no multitransmitter interference. As we have seen in Section II, in the slow fading case there are no tracking errors. For fast fading, where the 


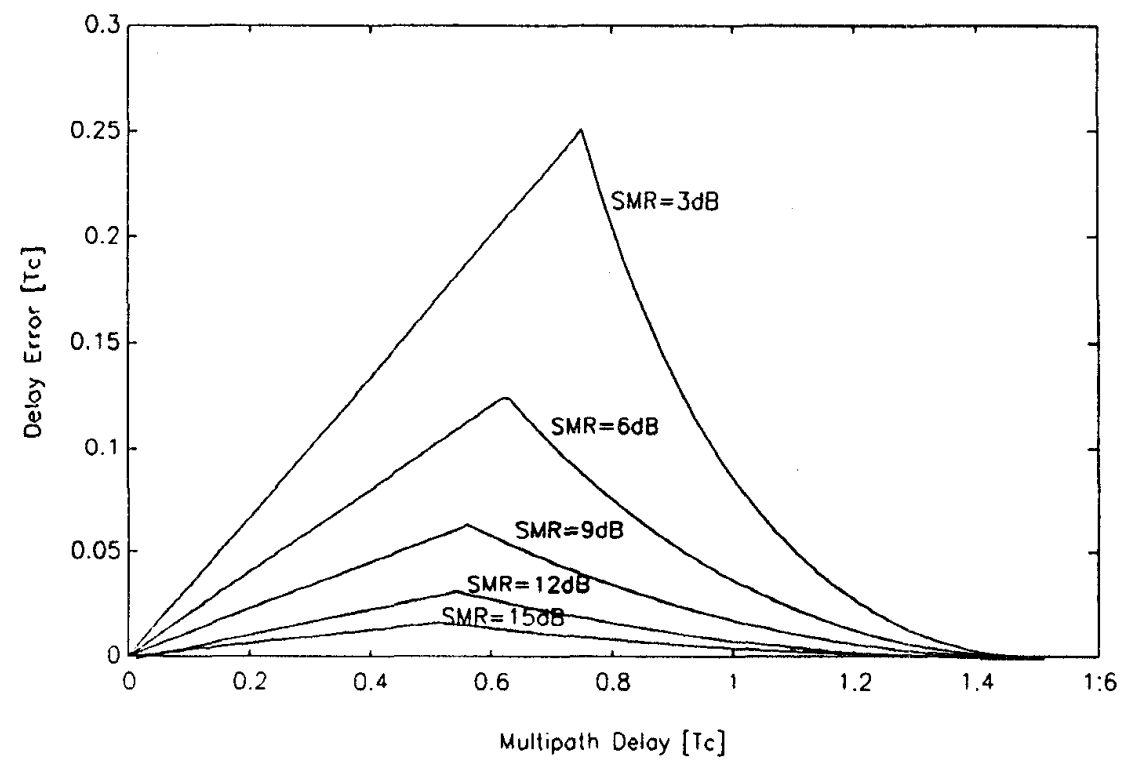

Fig. 2. Bias of a noncoherent DLL for early-late spacing: $d=1$.

estimated carrier phase equals the LOS phase, the correlation output can be written as

$$
z=a_{0} R(\hat{\tau})+a_{1} \cos \left(\theta_{1}\right) R\left(\hat{\tau}-\tau_{1}\right)
$$

Because the SMR is assumed to be greater than one, the peak of the correlation output has always the same delay as the peak of the correlation function of the LOS signal. So in this case, independent of the multipath delay and phase, the code delay that maximizes the correlation output is equal to the $\operatorname{LOS}$ delay $\left(\tau_{d}=0\right)$. For a noncoherent DLL, however, tracking biases are introduced. By determining the zero crossing of the $S$-curve with negative slope, for $d=1$ the resulting tracking bias $\tau_{e}$ (13) can be obtained from (4) with $T_{1}=0.5 T_{c}\left(a_{1}^{2} / a_{0}^{2}+1\right)$; see (13) at the bottom of the page. With the SMR as a parameter, the biases are depicted in Fig. 2 If the SMR is large enough, the tracking error is small. In the case of strong multipath signals, however, the tracking error can become considerable; for instance $\tau_{e}=0.25 T_{c}$ for $\mathrm{SMR}=3 \mathrm{~dB}$ and $\tau_{1}=0.75 T_{c}$.

From (12), we can see that for a certain multipath delay $\tau_{1}$, the correlation output will vary due to the varying multipath phase $\theta_{1}$. In Fig. 3, the instantaneous correlation output as a function of $\theta_{1}$ is shown for a multipath delay of $0.7 T_{c}$ and $\mathrm{SMR}=5 \mathrm{~dB}$. The correlation output is plotted for $\hat{\tau}=\tau_{e}$, the delay as estimated by the noncoherent DLL, and for $\hat{\tau}=0$, if the tracking bias is not taken into account and the correlation output is maximum. In the shown case, the loss of signal power due to the tracking error amounts to almost $3 \mathrm{~dB}$ in the out-ofphase situation. In the worst case situation (maximum delay error) for $\mathrm{SMR}=3 \mathrm{~dB}$, viz. $\tau_{1}=0.75 T_{c}$, it can be calculated that the tracking bias causes a loss of signal power of more than $6 \mathrm{~dB}$ for out-of-phase signals.

The loss of signal power results in a degradation of the bit-error rate (BER). The bit-error probability, given signal amplitude $A$ and gain/loss factor $x$, is represented by (7). The factor $x$ in this expression for $p_{e}$, is equal to the normalized ( $a_{0}=1$ ) correlation output (12), which is a function of the multipath phase $\theta_{1}$

$$
x\left(\theta_{1} \mid \tau_{1}\right)=R(\hat{\tau})+a_{1} \cos \left(\theta_{1}\right) R\left(\hat{\tau}-\tau_{1}\right) .
$$

Because we consider only one user, the variance of the Gaussian noise, $\sigma^{2}$, is just the noise variance $N_{0} / T_{b}$. With the bit energy-to-noise ratio is defined as $\frac{E_{b}}{N_{0}} \hat{=} \frac{A^{2} T_{b}}{2 N_{0}}$, for an uniform distributed multipath phase $\theta_{1}$, the bit-error probability given a multipath delay $\tau_{1}$, becomes

$$
P_{e, \tau_{1}}=\frac{1}{2 \pi} \cdot \int_{-\pi}^{\pi} \frac{1}{2} \operatorname{erfc}\left(x\left(\theta_{1} \mid \tau_{1}\right) \sqrt{\frac{E_{b}}{N_{0}}}\right) d \theta_{1} .
$$

Fig. 4 shows the bit-error probabilities for $t_{1}=0.7 T_{c}$ for a typical value of the SMR: $\mathrm{SMR}=5 \mathrm{~dB}$. In this plot, $P_{e}$ is drawn for the cases that the signal delay is estimated with and without code tracking bias, and for the multipath-free channel. It is obvious that the code tracking error results in a degradation of the bit-error probability.

If the bias is taken into account, to maintain a bit-error probability of $10^{-3}$, the SNR has to be increased by more than $2 \mathrm{~dB}$ as compared to the bias-free situation. So, given a bit-error probability, there is a loss in SNR due to the tracking

$$
\tau_{e}= \begin{cases}\frac{a_{1}^{2}}{a_{0}^{2}+a_{1}^{2}} \tau_{1}, & 0 \leq \tau_{1} \leq T_{1} \\ T_{c} \cdot\left(\frac{\tau_{1}}{T_{c}}-1.5+\frac{a_{0}^{2}}{a_{1}^{2}}-\sqrt{\frac{a_{0}^{2}}{a_{1}^{2}}\left(2 \frac{\tau_{1}}{T_{c}}-3+\frac{a_{0}^{2}}{a_{1}^{2}}\right)}\right), & T_{1} \leq \tau_{1} \leq 1.5 T_{c}\end{cases}
$$




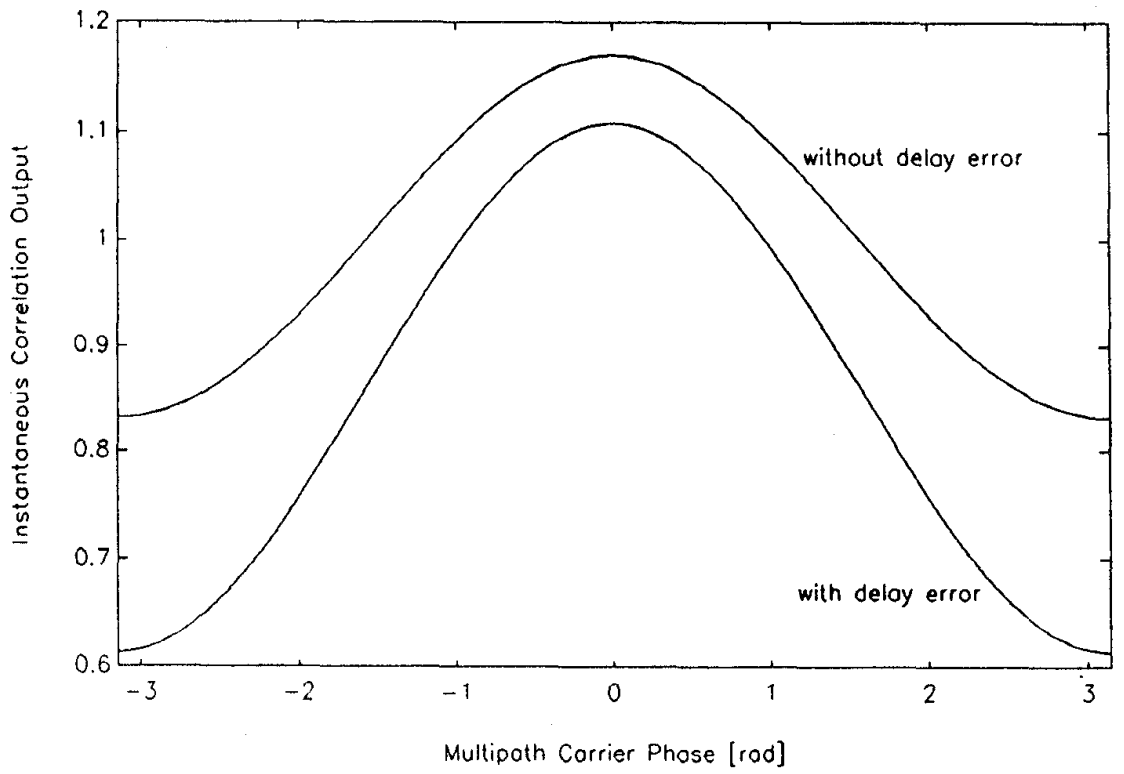

Fig. 3. Instantaneous correlation output as a function of the multipath carrier phase $\theta_{1}$, for SMR $=5 \mathrm{~dB}$, multipath delay: $\tau_{1}=0.7 T_{c}$ and early-late spacing: $d=1$.

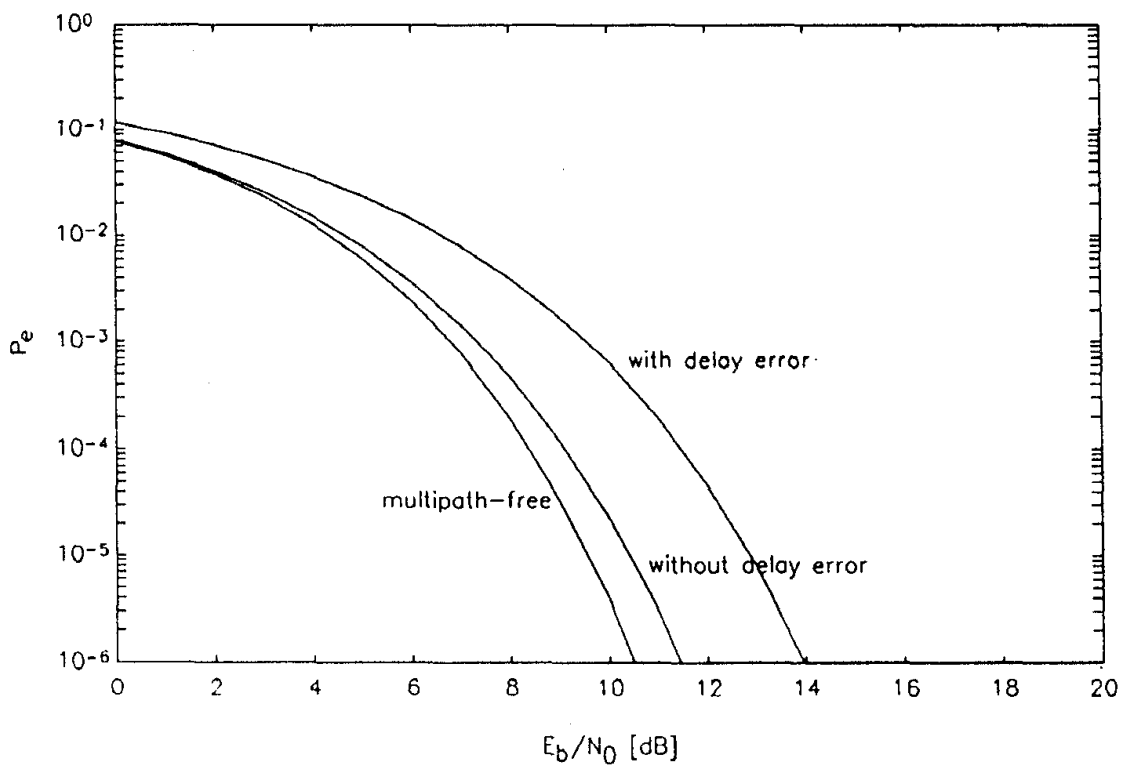

Fig. 4. Bit-error probability without delay error, with delay error and in the multipath-free case, for $\mathrm{SMR}=5 \mathrm{~dB}, d=1$, and $\tau_{1}=0.7 T_{c}$.

bias. For $P_{e}=10^{-3}$, the increase in SNR as compared to the situation where the code delay error is ignored, is depicted in Fig. 5. In the case of the maximum delay error for SMR = $3 \mathrm{~dB}\left(\tau_{1}=0.75 T_{c}\right)$, the SNR has to be increased by about $5.5 \mathrm{~dB}$.

To visualize the degradation of the BER due to the tracking bias, for a SNR of $10 \mathrm{~dB}$, the bit-error probability calculated with bias divided by the bit-error probability without bias is plotted versus the multipath delay $\tau_{1}$ (Fig. 6), with SMR as a parameter. This ratio of the bit-error probability calculated with bias and the bit-error probability without bias is called the degradation ratio. For a SMR of $3 \mathrm{~dB}$ and a multipath delay of $0.75 T_{c}$, the bit-error probability calculated with delay bias is even 300 times larger than the probability if the tracking error is not taken into account.

\section{B. Multiple Multipath Signals}

Now we have gained insight into the influence of code tracking errors on the bit-error probability in the situation of an input signal consisting of a LOS component and just one multipath signal, we extend our analysis to multiple multipath signals and more performance parameters. Again, we consider unfiltered signals. 


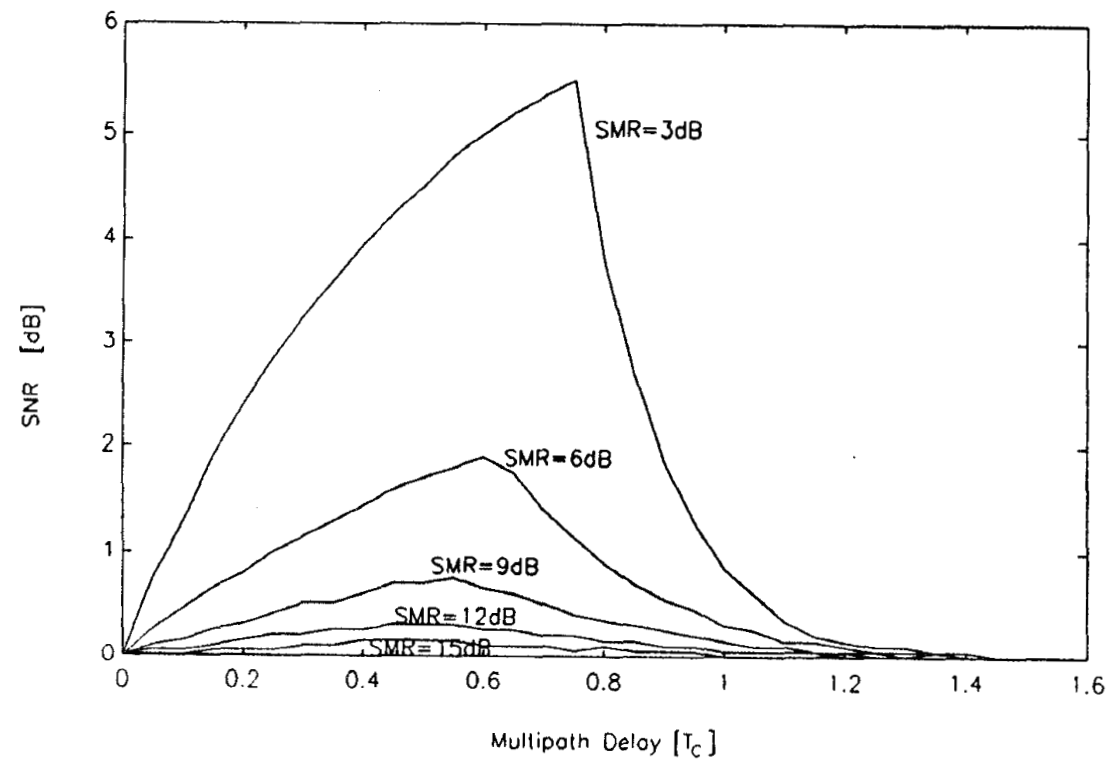

Fig. 5. SNR increase to maintain a bit-error probability of $10^{-3}$ as compared to the situation where the code delay error is ignored for $d=1$.

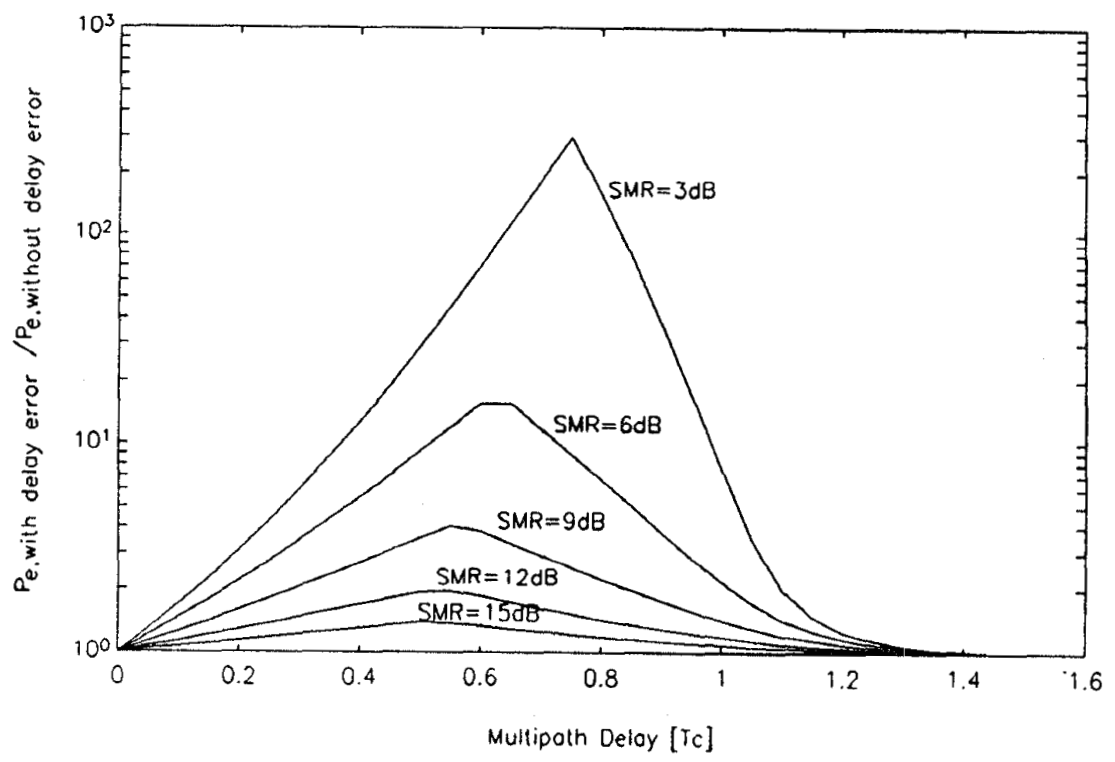

Fig. 6. $P_{\varepsilon}$ obtained with delay error divided by $P_{e}$ obtained without delay error for $E_{b} / N_{0}=10 \mathrm{~dB}$ and $d=1$.

To model the multipath signals we will use the power-delay profile. For rural and suburban environments, the average power-delay profile of multipath signals is approximately exponential [14], [15]

$$
P(\tau)=\frac{1}{T_{s}} b_{0} \exp \left(-\frac{1}{T_{s}} \tau\right)
$$

where $T_{s}$ is the delay spread. For a rural environment, a typical value of $T_{s}$ is $0.65 \mu \mathrm{s}$ [15]. As for the situation of one multipath signal, we consider multipath signals with a delay of up to $1.5 T_{c}$. If we take $M_{\text {chip }}$ signals between $\tau=0$ and $\tau=T_{C}$, so the total number of multipath signals $M$ will be $1.5 \cdot M_{\text {chip }}$, the power-delay profile can be used to calculate the average multipath power for each signal. For signal number $m$, the multipath power $b_{m 0}$ is approximated as the power between the delay values $m \cdot T_{c} / M_{\mathrm{chip}}$ and $(m+1) \cdot T_{c} / M_{\mathrm{chip}}$

$$
b_{m 0}=b_{0}\left[1-\exp \left(-\frac{T_{c}}{M_{\mathrm{chip}} T_{s}}\right)\right] \exp \left[-m \frac{T_{c}}{M_{\mathrm{chip}} T_{s}}\right] .
$$

By knowing the average multipath power for each signal, the $S$-curve (4) can be calculated. Determining the zero crossing of the $S$-curve with negative slope gives the tracking bias, $t_{\mathrm{Bias}}$, of the noncoherent DLL in a fast fading environment. In Fig. 7, the biases are depicted with SMR as a parameter for $M_{\text {chip }}=10$ and $d=1$. For large values of $T_{s} / T_{c}$ the tracking bias becomes negligible: if spread-spectrum modulation is 


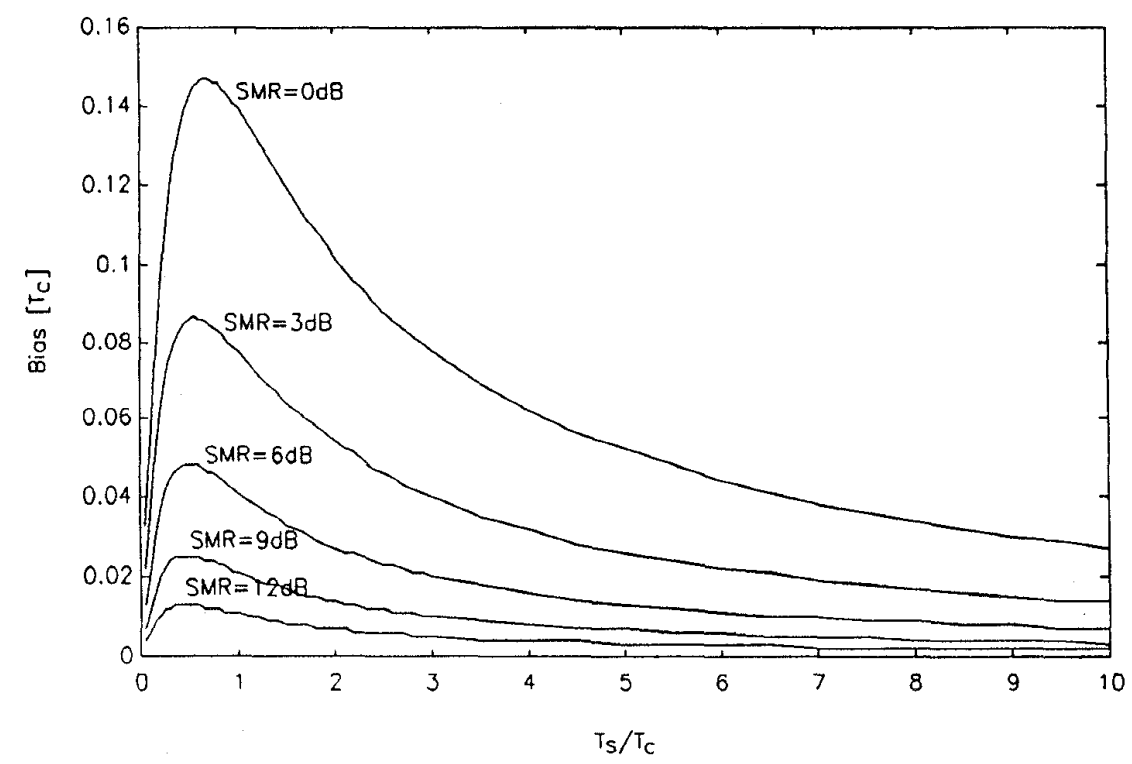

Fig. 7. Bias of a noncoherent DLL for $M=15$ and $d=1$.

used with a chip time $T_{c}$ that is less than the delay spread $T_{s}$ of the channel, then the multipath power is partially reduced by the correlation operation in the receiver.

Notice that the bias is not equal to the instantaneous delay error, $\hat{\tau}-\tau_{d}$. Due to the varying multipath phases $\theta_{i}$, the code delay that maximizes the correlation output is not equal to the line-of-sight delay $\left(\tau_{d}=0\right)$. Because it is impractical to determine the instantaneous delay error and because the code tracking error is caused mainly by the bias, from now on only the tracking bias is considered.

To calculate the bit-error probability $(6), p_{u}(x)$, the probability density function (PDF) of the desired part of the correlation output $z$, has to be known. Since the received signal can be considered as the sum of a LOS signal and a number of multipath signals with random varying phases, the correlation output can be described by a Gaussian PDF. This assumes that the fading bandwidth is large in comparison with the tracking loop bandwidth of the code and carrier tracking loops, which causes the receiver to track the average vector component (being the LOS signal) whereas the multipath components, multiplied by the cosine of a random phase, will contribute a Gaussian component to the correlation output. The mean of the PDF is equal to the amplitude of the LOS signal times the output of the correlation function of the LOS signal: $a_{0} \cdot R_{\operatorname{LOS}}(\hat{\tau})$, the variance of the Gaussian PDF is determined by the residue of the multipath power after the correlation operation in the receiver and is, therefore, also depending on the estimated code delay $\hat{\tau}$.

To obtain the bit-error probability given $A$ and $x, p_{e}(x A)$ (7), the total variance of the Gaussian noise, which consists of the noise variance $N_{0} / T_{b}$ and the interference power $\sigma_{i}^{2}$, has to be determined. In [3], a closed form expression was derived for the variance $\sigma_{i}^{2}$ of $K$ interfering products. When lognormal shadowing is not taken into account, the normalized equation is

$$
\sigma_{i}^{2}=\frac{2 K}{3 N}\left(b_{0}+\frac{1}{2}\right) .
$$

Here, $N$ is the length of the spreading code and $b_{0}$ is the average scattered power due to multipath.

To gain insight into the effects of the tracking bias on the bit-error probability, first, we consider the situation for one user. We have compared the bit-error probability for the case where the tracking bias is ignored $\left(\hat{\tau}=\tau_{0}\right)$ with the bit-error probability for the case where the tracking bias is taken into account $\left(\hat{\tau}=\tau_{\text {Bias }}\right)$. The bit-error probabilities are shown for two $T_{s} / T_{c}$ values, viz. 0.65 and 6.5 , in Fig. 8 for $\mathrm{SMR}=6 \mathrm{~dB}$.

For $T_{s} / T_{c}=6.5$, the bit-error probability degradation is small, for $T_{s} / T_{c}=0.65$, however, the degradation is considerable. As we can see in Fig. 8, for $E_{b} / N_{0}=10 \mathrm{~dB}$ the bit-error probability obtained with bias is 4.6 times as worse as the bit-error probability obtained without bias; for $E_{b} / N_{0}=$ $20 \mathrm{~dB}$ it is almost 21 times larger. If the bias is taken into account, to maintain a bit-error probability of $10^{-3}$ and $10^{-4}$, the SNR has to be increased by about $3 \mathrm{~dB}$ and by almost 7 $\mathrm{dB}$, respectively, as compared to the bias-free situation.

To determine the throughput and delay, multitransmitter interference is taken into account. For constant values of $E_{b} / N_{0}$ and $N$ and with SMR and $T_{s} / T_{c}$ as parameters, (6), (7), and (18) are used to calculate the bit-error probability as a function of the number of interfering products $K$. With (8) to obtain the packet success probability $P_{s}$ and (9) to obtain the PDF of the offered traffic $P_{\mathrm{tr}}$, (10) yields the throughput $S$. The following results are obtained for $E_{b} / N_{0}=20 \mathrm{~dB}$, $N=63$ chips per bit, $N_{p}=256$ bits per packets, $t=10$, $K_{\max }=2 \mathrm{~N}$ users, $\mathrm{SMR}=6 \mathrm{~dB}$, and for two $T_{s} / T_{c}$ ratios, viz., 0.65 and 6.5 .

Fig. 9 shows the normalized throughput, $S / N$, curves for the situation where the tracking bias is ignored and the situation where the bias is taken into account. 


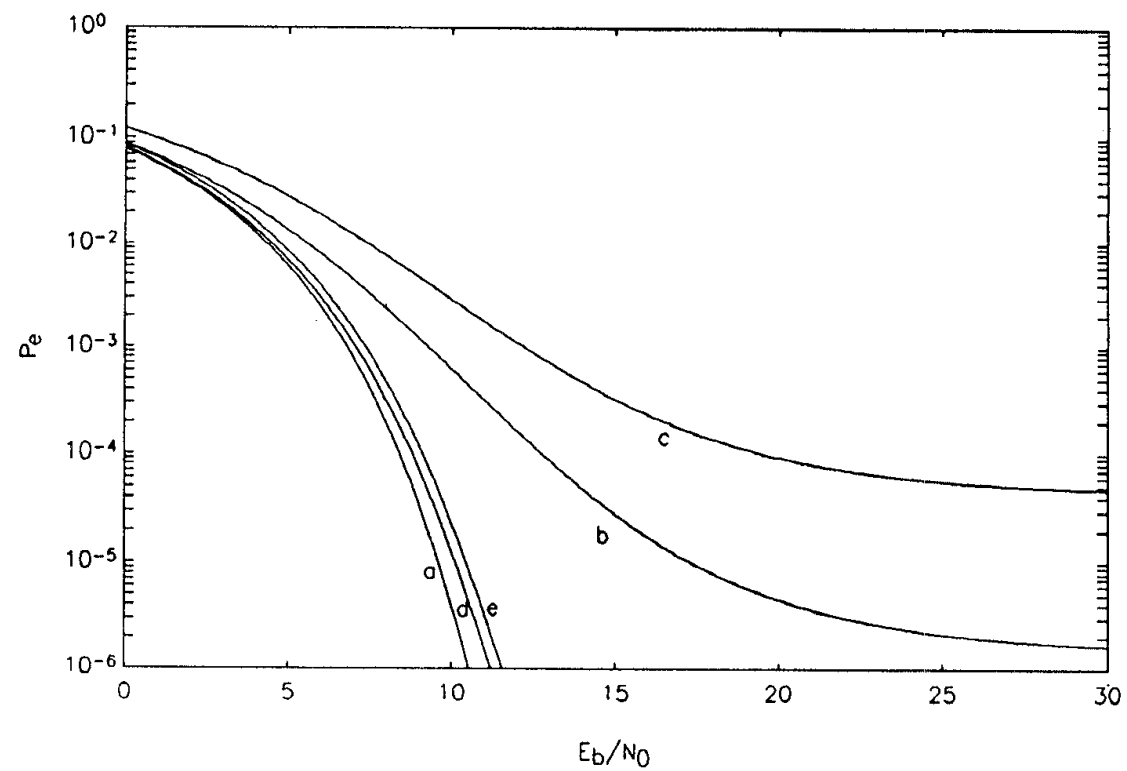

Fig. 8. Bit-error probability for SMR $=6 \mathrm{~dB}$ : (a) multipath free, (b) without bias, $T_{s} / T_{c}=0.65$, (c) with bias, $T_{s} / T_{c}=0.65$, (d) without bias, $T_{s} / T_{c}=6.5$, and (e) with bias, $T_{s} / T_{c}=6.5$.

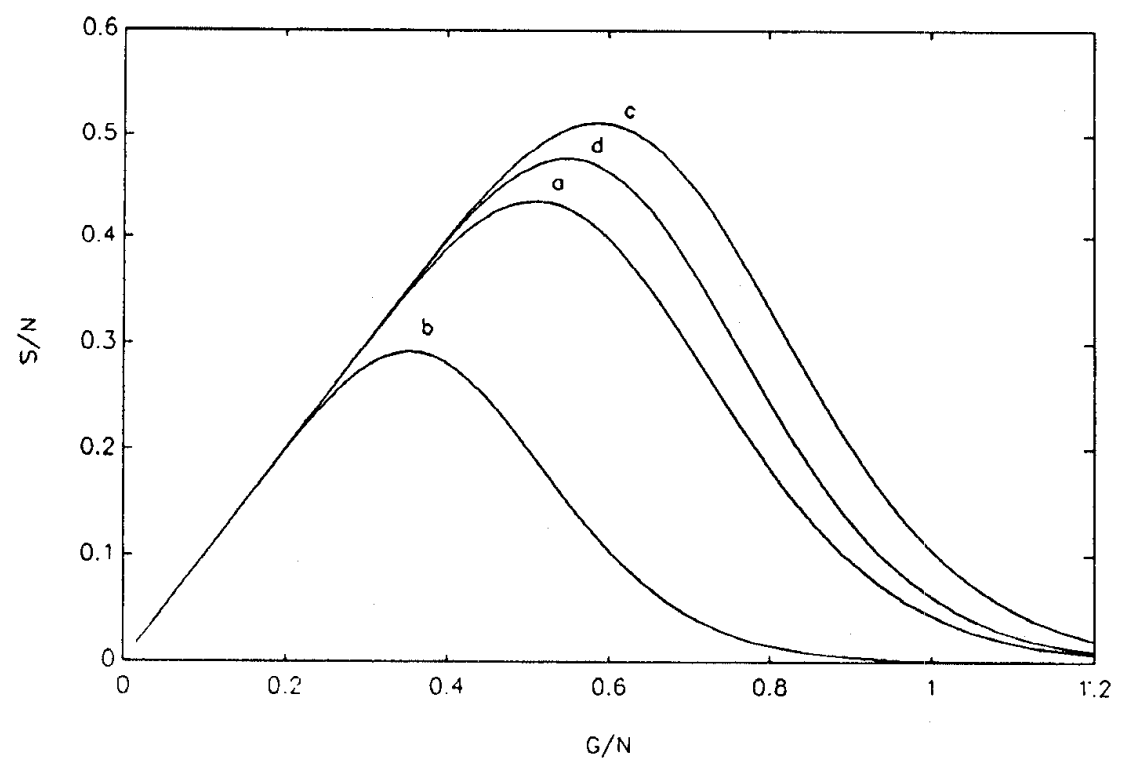

Fig. 9. Normalized throughput of slotted CDMA for SMR $=6 \mathrm{~dB}$ : (a) without bias, $T_{s} / T_{c}=0.65$, (b) with bias, $T_{s} / T_{c}=0.65$, (c) without bias, $T_{s} / T_{c}=6.5$, and (d) with bias, $T_{s} / T_{C}=6.5$.

As we would expect from the bit-error probability degradation, the degradation of the throughput performance due to the tracking bias is larger for a $T_{s} / T_{c}$ ratio of 0.65 than for a ratio of 6.5. Fig. 9 demonstrates that for SMR $=6 \mathrm{~dB}$ and $T_{s} / T_{c}=0.65$ the maximum throughput is more than 1.5 times too optimistic, if the tracking bias is ignored. Computational results showed that for $S M R=3 \mathrm{~dB}$, this result is almost three times too high.

Finally some results on the delay are given, obtained using (11) with $N_{\mathrm{AT}}=3$ and $T_{d}=74$ slots. In Fig. 10, the normalized throughput-delay curves for the cases with and without tracking bias are compared. Again the results show a considerable degradation of the performance in the case of a delay bias, especially for $T_{s} / T_{c}=0.65$.

\section{POSSIBLE REMEDIES}

As explained in Section II, in practice, it is not possible to choose the tracking loop bandwidth and the data bandwidth of a system using a noncoherent DLL in such a way, that tracking errors can be avoided for all applications. To reduce the loss of signal power and the degradation of the performance due to the tracking bias, the early-late spacing could be decreased. In 


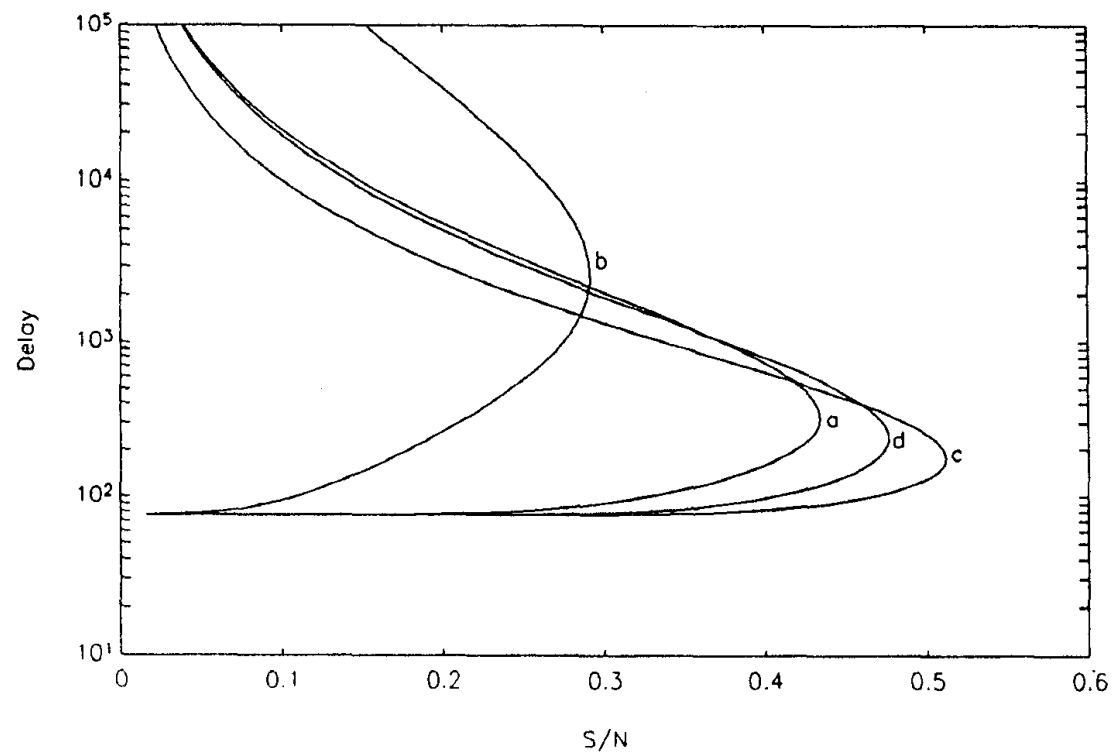

Fig. 10. Normalized throughput-delay curves of slotted CDMA for SMR $=6 \mathrm{~dB}$ : (a) without bias, $T_{s} / T_{c}=0.65$, (b) with bias, $T_{s} / T_{c}=0.65$. (c) without bias, $T_{s} / T_{c}=6.5$, and (d) with bias, $T_{s} / T_{c}=6.5$.

[10], it is shown that a smaller early-late spacing can result in a smaller tracking bias and thus in a smaller bit-error probability. However, this only helps if the spread-spectrum bandwidth is equal to or larger than $1 / d T_{c}$. In practice, many spreadspectrum communication systems use bandlimited signals with a double-sided bandwidth of about $2 / T_{c}$. For these systems decreasing the early-late spacing further than half a chip does not help. Although this will give a considerable improvement over an early-late spacing of one chip, the degradation will still be considerable.

Because it applies no squaring operations, a coherent DLL has no tracking bias for large $B_{F} / B_{L}$ values. For situations where fast fading can be expected, this type of DLL is preferable in order to minimize code tracking errors and, therefore, the bit-error probability degradation. Further, it is possible to greatly reduce code synchronization errors by using special tracking loops which are designed to deal with multipath or spread-spectrum interference, like described in [12] and [13].

\section{CONCLUSION}

If the tracking bias is not taken into account, the performance analysis (in terms of bit-error probability, throughput and delay) of a CDMA system or network shows too optimistic results in some situations. It is shown for noncoherent DLL receivers with an early-late spacing of $d=1$, that dependent on the received multipath power and the delay spread, the system performance can degrade significantly when tracking errors are taken into account. In practice, performance degradation due to the tracking bias of a noncoherent delay lock loop cannot always be avoided by system design. To overcome the tracking problems, other delay lock techniques can be applied, like the coherent DLL and special tracking loops which are designed to deal with multipath or spread-spectrum interference [12], [13].

\section{REFERENCES}

[1] M. B. Pursley, "Performance evaluation for phase-coded spreadspectrum multiple-access communication-Part I: System analysis," IEEE Trans. Commun., vol. 25, no. 8, pp. 795-799, Aug. 1977.

[2] R. D. J. van Nee, H. S. Misser, and R. Prasad, "Direct-sequence spread spectrum in a shadowed Rician fading land-mobile satellite channel," IEEE J. Select. Areas. Commun., vol, 10, pp. 350-357, Feb. 1992.

[3] R. D. J. van Nee and R. Prasad, "Spread-spectrum path diversity in a shadowed Rician fading land-mobile satellite channel," IEEE Trans. Veh. Technol., vol, 42, no. 2, pp. 131-136, May 1993.

[4] C. A. F. J. Wijffels, H. S. Misser, and R. Prasad, "A micro-cellular CDMA system over slow and fast Rician fading radio channels with forward error correcting coding and diversity," IEEE Trans. Veh. Technol., vol. 42 , no. 4, pp. $570-580$, Nov. 1993.

[5] B. R. Vojcic, R. L. Pickholtz, and L. B. Milstein, "Performance of DSCDMA with imperfect power control operating over low earth orbiting satellite link," IEEE J. Select. Areas Commun., vol. 12, no. 4, pp. 560-567, May 1994.

[6] A. K. Elhakeem, R. DiGirolamo, I. B. Bdira, and M. Talla, "Delay and throughput characteristics of TH, CDMA, TDMA, and hybrid networks for multipath faded data transmission channels," IEEE J. Select. Areas. Commun., vol. 12, no. 4, pp. 622-637, May 1994.

[7] R. D. J. van Nee, R. N. van Wolfswinkel, and R. Prasad, "Slotted ALOHA and code division multiple access techniques for land-mobile satellite personal communications," IEEE J. Select. Areas. Commun., vol. 13, no. 2, pp. 382-388, Feb. 1995.

[8] M. G. Jansen and R. Prasad, "Capacity, throughput, and delay analysis of a cellular DS CDMA system with imperfect power control and imperfect sectorization," IEEE Trans. Veh. Technol, vol. 44, no. 1, pp. 67-75, Feb. 1995.

[9] R. Prasad, H. S. Misser, and A. Kegel, "Performance evaluation of direct-sequence spread spectrum multiple-access for indoor wireless communication in a Rician fading channel," IEEE Trans. Commun., vol. 43, nos. 2/3/4, pp. 581-592, Feb./Mar./Apr. 1995.

[10] R. D. J. van Nee, "Spread-spectrum code and carrier synchronization errors caused by multipath and interference," IEEE Trans. Aerosp. Electron. Syst., vol. 29 , no. 4, pp. 1359-1365, Oct. 1993.

[11] W. J. Weber, "Performance of phase-locked loops in the presence of fading communication channels," IEEE Trans. Commun., vol. 24, pp. 487-499, May 1976.

[12] R. D. J. van Nee, "The multipath estimating delay lock loop," in Proc. IEEE 2nd Int. Symp. Spread Spectrum Techniques Applicat., Yokohama, Nov. 29-Dec. 2, 1992, pp. 39-42.

[13] __ "Multipath and multitransmitter interference in spread-spectrum communication and navigation systems," Ph.D. dissertation, Delft University of Technology, The Netherlands, 1995. 
[14] W. C. Y. Lee, Mobile Communications Engineering. New York: McGraw-Hill, 1982.

[15] J. van Rees, "Measurements of the wide-band radio channel characteristics for rural, residential and suburban areas," IEEE Trans. Veh. Technol., vol. 36, pp. 2-6, Feb. 1987.

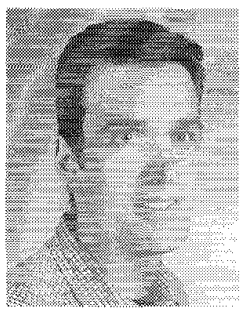

Bas W.'t. Hart ( $\left.S^{\prime} 95\right)$ was born in Krimpen a/d IJssel, The Netherlands, on February 19, 1970. He received the M.Sc. degree in electrical engineering from Delft University of Technology, Delft, The Netherlands, in 1994.

After his graduation, he joined the Telecommunications and Traffic-Control Systems Group of the Electrical Engineering Department of Delft University of Technology as a post-graduate student, where he is working toward the degree of Chartered Designer in the field of information technology.

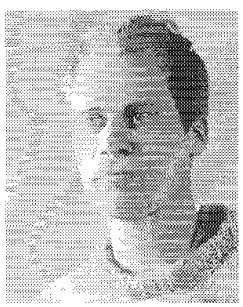

Richard D. J. van Nee (M'95) was born in Schoonoord, The Netherlands, on January 17, 1967. He received the M.Sc. degree in electrical engineering (cum laude) and the Ph.D. degree (cum laude) from Twente University, The Netherlands, in 1990 and 1995 , respectively.

His Ph.D. research was on the effects of multipath and multiuser interference on spread-spectrum communication and navigation systems. From January 1995, he worked as a Private Consultant to Nov Atel Communications, Calgary, working on the design of multipath-resistant GPS receivers for high precision applications. In March 1995, he joined AT\&T Bell Laboratories, Utrecht, where he is working in the area of high-speed wireless communications.

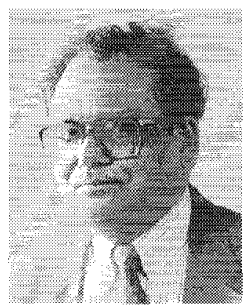

Ramjee Prasad (M'88-SM'90) was born in Babhnaur (Gaya), Bihar, India, on July 1, 1946. He received the B.Sc. (Eng.) degree from the Bihar Institute of Technology, Sindri, India, and the M.Sc. (Eng.) and Ph.D. degrees from the Birla Institute of Technology (BIT), Ranchi, India, in 1968, 1970, and 1979, respectively.

In 1970, he joined BIT as Senior Research Fellow and became an Associate Professor in 1980. While he was with BIT, he supervised many research projects in the area of microwave and plasma engineering. From 1983 to 1988 , he was with the University of Dar es Salaam (UDSM), Tanzania, where he became Professor in Telecommunications at the Department of Electrical Engineering in 1986. At UDSM, he was responsible for the collaborative project "Satellite Communications for Rural Zones" with Eindhoven University of Technology, The Netherlands. Since February 1988, he has been with the Telecommunications and Traffic Control Systems Group, Delft University of Technology (DUT), The Netherlands, where he is actively involved in the area of personal, indoor, and mobile radio communications (PIMRC). He is Head of the Transmission Research Section of IRCTR (International Research Centre for Telecommunications-Transmission and Radar). He is currently involved in the European ACTS project FRAMES (Future Radio Wideband Multiple Access System) as a Project Leader of DUT. He has published over 200 technical papers. His current research interest lies in wireless networks, packet communications multiple-access protocols, adaptive equalizers, spread-spectrum CDMA systems, and multimedia communications. He has served as a member of advisory and program committees of several IEEE international conferences. He has also presented keynote speeches, invited papers, and tutorials on PIMRC at various universities, technical institutions, and IEEE conferences. He was the Organizer and Interim Chairman of the IEEE Vehicular Technology/Communications Society Joint Chapter, Benelux Section. He is now the Elected Chairman of the joint chapter. $\mathrm{He}$ is also founder of the IEEE Symposium on Communications and Vehicular Technology (SCVT) in the Benelux and he was the Symposium Chairman of SCVT93. He is the Coordinating Editor and Editor-in-Chief of the Kluwer international journal on Wireless Personal Communications and also a member of the editorial board of other international journals, including the iEEE COMmunications Magazine and the IEE Electronics COMmUNication Engineering Journal. He was the Technical Program Chairman of the PIMRC'94 International Symposium held in The Hague, The Netherlands, during September 19-23, 1994, and also of the Third Communication Theory Mini-Conference in conjunction with GLOBECOM'94 held in San Francisco, CA, November 27-30, 1994.

Dr. Prasad is listed in the US Who's Who in the World. He is a fellow of the IEE, a Fellow of the Institution of Electronics \& Telecommunication Engineers, a Member of the New York Academy of Sciences, and of NERG (The Netherlands Electronics and Radio Society). 